\title{
1048 - Sectioning of Cultured Cells by Ar Ion Beam Milling for SEM Observations
}

Shigeyasu Tanaka and Yusuke Ohmi

Chubu University, Kasugai, Aichi, Japan

TEM has been used for cross-sectional observations of biological samples by electron microscopy. However, the sample preparation for TEM observations requires laborious procedures and takes from several hours to a day or more. The sample preparation for SEM observations is less laborious, thus SEM is used more and more for cross-sectional observations. As a sectioning technique for biological samples, focused ion beam (FIB) has received much attention [1], because it has been used in material science and industry fields to obtain accurately and precisely sectioned samples. But, because of the high beam energy, ion-beam induced damage is more severe in the biological field, and precautions are vitally necessary. In a previous paper [2], we have reported an alternative technique for sectioning of cells, which is based on low-energy Ar ion-beam milling, and is easy and rapid. Here, we report the details of this method for obtaining good samples using HEK293T as a sample.

A silicon plate was coated by $0.1 \mathrm{mg} / \mathrm{mL}$ poly-L-lysine in phosphate buffered saline (PBS) for $3 \mathrm{~h}$ at room temperature. The coated silicon plate was washed by PBS. HEK293T cell $\left(1.4 \times 10^{6} \mathrm{cell} / \mathrm{ml}\right)$ was cultured with $10 \%$ FCS containing DMEM medium on the poly-L-lysine coated silicon plate overnight at $37^{\circ} \mathrm{C}$ in $5 \% \mathrm{CO}_{2}$ incubator. Then the cell on silicon was fixed with $3 \%$ glutaraldehyde solution for $2 \mathrm{~h}$. After washing with PBS, the sample was stained with Platinum Blue. Then the sample was processed by BEL1 (Nisshin EM). After this treatment, the sample was dried in a vacuum chamber. Then the silicon plate was cut into a rectangular shape. In this process, sharp edges were created by cleavage. The sharp edge was used as a shield. Finally, the rectangular silicon piece was fixed to a sample holder of Ion Slicer (JEOL), and Ar ion beam was irradiated. The ion irradiation was carried out in such a way that most of cells is in the shadow of the silicon plate with respect to the Ar ion beam (Fig. 1(a)). The diameter of the ion beam was about $1 \mathrm{~mm}$. The ion beam currents were about $20 \mu \mathrm{A}, 30 \mu \mathrm{A}$ and $50 \mu \mathrm{A}$ for accelerating voltages of $1 \mathrm{kV}, 2 \mathrm{kV}$ and $3 \mathrm{kV}$, respectively. SEM observations were performed at $3 \mathrm{kV}$. An example of the SEM image is shown in Fig. 1(b).

In the course of the sectioning experiments, we noticed that the edge of the Si plate was sputtered gradually, and the quality of the sectioned face was strongly dependent on the ion accelerating voltage. To show this clearly, experiments with varying milling conditions were carried out as shown in Fig. 2. Fig. 2(a) is an SEM image of an as-cleaved sample. The angle $\theta$ is about $12^{\circ}$ for this sample. The cleaved facet appears bright. Fig. 2(b) is the SEM image of the sample milled with $2 \mathrm{kV}$ for 30 min. The Si edge is seen to be sputtered between the arrows as dark. The upper part of the cell left unsputtered. To remove this part, an additional milling with $3 \mathrm{kV}$ for $5 \mathrm{~min}$, followed by $2 \mathrm{kV}$ for $5 \mathrm{~min}$ was carried out (Fig. 2(c)). Now, the whole cross-section is seen. Fig. 2(d) is the result of the additional milling with $2 \mathrm{kV}$ for $10 \mathrm{~min}$, from the sample of Fig. 2(c). Sectioning of the cell and sputtering of the Si edge proceed, as milling continues. Fig. 2(e) is the result of the additional milling with $1 \mathrm{kV}$ for $10 \mathrm{~min}$, from the sample of Fig. 2(d). Now, the cross-section of the cell appears brighter, indicating a sign of charging. This feature disappears after additional milling with $2 \mathrm{kV}$ for $10 \mathrm{~min}$, as shown in Fig. 2(f). It should be mentioned that the cross-section showed a streak pattern along the ion beam when milled with $3 \mathrm{kV}$ (not shown). Milling with $2 \mathrm{kV}$ was effective to eliminate such a pattern and to obtain flat surface [3]. 
(a)

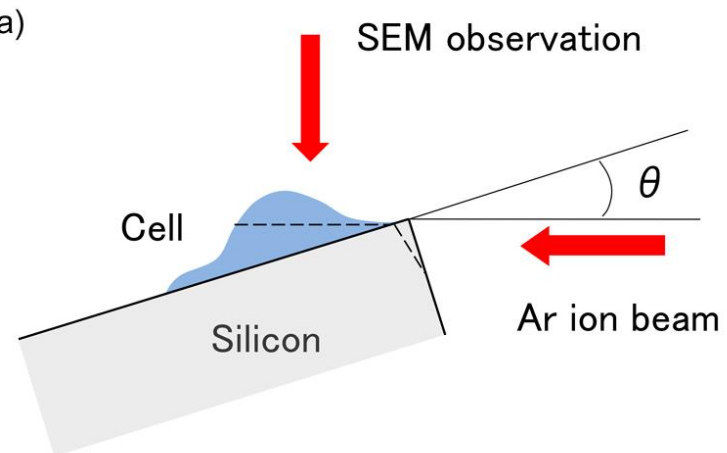

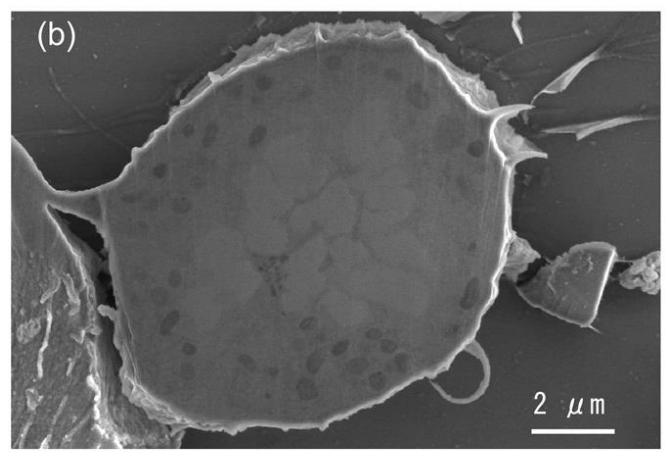

Figure 1. (a) Schematic representation of sectioning process and SEM observation direction. Cell is on the Si surface. The Si surface is inclined with respect to the Ar ion beam. Dotted line indicates a milled surface. (b) An example of the SEM image.
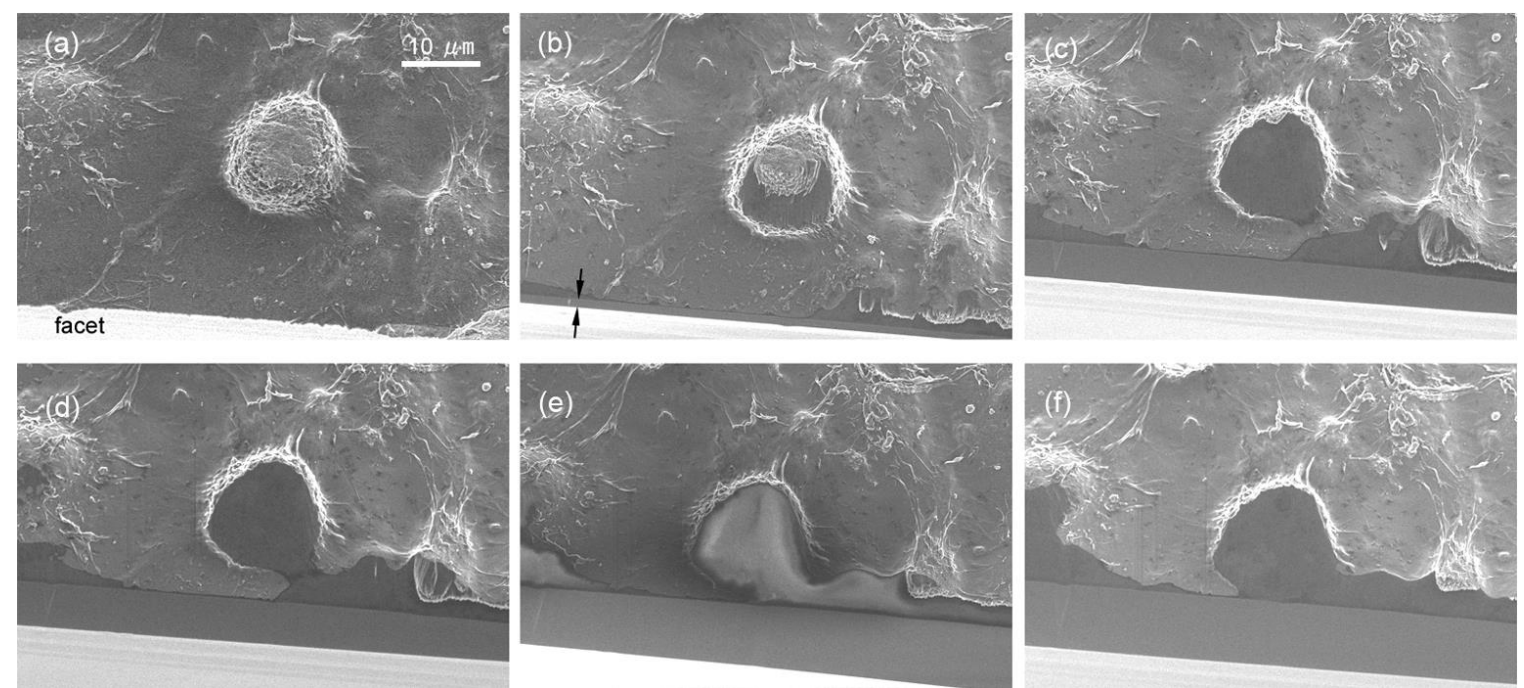

Figure 2. SEM images of a sample milled with various conditions. (a) As-cleaved. (b) After milling with $2 \mathrm{kV}$ for $30 \mathrm{~min}$. (c) After additional milling with $3 \mathrm{kV}$ for $5 \mathrm{~min}$, followed by $2 \mathrm{kV}$ for $5 \mathrm{~min}$, from the sample of (b). (d) After additional milling with $2 \mathrm{kV}$ for $10 \mathrm{~min}$, from the sample of (c). (e) After additional milling with $1 \mathrm{kV}$ for $10 \mathrm{~min}$, from the sample of (d). (f) After additional milling with $2 \mathrm{kV}$ for $10 \mathrm{~min}$, from the sample of (e).

\section{References}

[1] H. L. Hing et al, Microsc Microanal 13(Suppl 2) (2007) p. 1528.

[2] S. Tanaka and Y. Ohmi, Microsc Microanal 25(Suppl 2) (2019) p. 902.

[3] This work was supported in part by JSPS KAKENHI Grant Number JP18K04246. 